\section{GEOLOGY AND THE FUTURE OF COAL SUPPLIES}

$I^{\mathrm{N}}$ $\mathrm{N}$ the presidential address to Section C (Geology) of the British Association, delivered at Dundee on September 1, Dr. Murray Macgregor stressed the vital need for closer co-operation between the miner and the geologist for the better-planned exploitation of the coal resources of Great Britain; his address was followed by a discussion on the future prospects of those supplies.

In opening the discussion, Prof. H. G. A. Hickling suggested the importance of attention to the various classes of coal and their most effective use, rather than the estimation of total reserves. Even with the wasteful usage of former years, the total supply is unlikely to approach exhaustion within any period for which it is reasonable to plan at this time; but it is less certain that one can be equally optimistic regarding some of the most valuable varieties. The first-class coking-coals, the anthracites and some other classes have hitherto been obtainable only from very limited areas in Great Britain, where they have been heavily wrought and the reserves greatly reduced. It is consequently a matter of major importance whether the remaining reserves are likely to yield a greater or smaller proportion of such types. Any useful forecast must be based on a knowledge of the distribution of existing coal types and its causes.

Coals vary in their characteristics from two main causes : first, from variation in the original deposit in respect of the contributory plant material, its mode and extent of decay and the nature and quantity of associated mineral matter ; and secondly, as the result of the varying extent of subsequent alteration under geological influences. Regarding the first, the long-familiar division of coals into 'brights' and 'hards' corresponds broadly to those ('brights') which have been formed mainly from pieces of organically saturated wood and bark, in contrast with the 'hards', which consist of a far more highly disintegrated mass of the most resistant plant residues, forming a minute micro-conglomerate. Chemical examination indicates that the substance of the bright coal was originally of relatively constant composition. The duller component is extremely variable both in visible constitution and in chemical characteristics.

Most seams contain a mixture of the two principal types of material. Their varying proportions, and especially the constitution of the dull coal, give rise to the characteristics of the different seams. The extensive investigations carried out by the Fuel Survey in Great Britain during the past thirty years have shown that individual seams commonly maintain their special features over considerable areas, while all the seams in any field usually show similar changes in 'rank' as they are traced from one part of the field to another. These latter changes are most familiarly indicated by alteration of the percentage of 'volatile' yielded by the coal on heating; this diminishing progressively from the low-rank bituminous coals through the gas-coals, coking-coals and steam-coals to the highest grade anthracites.

Whether the variation of rank in any field be great or small, it follows the same pattern in all seams. Similar changes of rank are found in the vertical sequence of seams at any given point, though this very significant fact is commonly obscured by the individual characteristics of the seams unless a long vertical series can be examined. The rank rises with increasing depth, and accumulating evidence indicates considerable regularity in the rate of change. Our growing knowledge of the causes of the differences among coals leads to the possibility of a more accurate forecast of the probable quality of our remaining resources, and to the conclusion that, while we shall be compelled to work at greater depths, there will be some compensation from the inclusion of a measurably greater proportion of the higher-rank coals.

Dr. C. A. Seyler described his recent investigations into the mode of change of the main constituent (vitrains) of normal coals, as indicated by their increase of reflecting power as the coal advances in rank. Contrary to expectation, this alteration appears to be discontinuous, the reflecting power changing abruptly through a series of nine steps in geometrical progression, and terminating with the highest member, fusinite. In any coal the bulk of the vitrain belongs to one of the lower members, with smaller quantities of the next two or three and usually a little fusinite. As the rank of the seam advances to a critical point, each component present changes its reflecting power (and presumably its composition) one step upward in the series ; except, of course, the fusinite.

Exactly similar stepwise changes of the vitrinites are observed in coals which have been subjected to progressive alteration by the thermal metamorphism of sills and dykes. This is shown with exceptional completeness in the case of a Sumatra lignite, which has been changed progressively to an anthracitic condition through a series of stages closely corresponding with normal coals. It seems to afford conclusive proof that heat (and possibly pressure) is capable of accounting for the varying stages of alteration of normal coals.

Dr. A. C. Monkhouse gave an account of the work and results of the Physical and Chemical Survey of Coal Resources during the past twenty-five years by the nine coalfield survey laboratories and the Fuel Research Station at Greenwich. This has given a knowledge of the coal resources of Great Britain more complete than that of any other country in the world. Most of the important seams in all the principal fields have been examined in detail in many places. Analysis band by band has frequently shown how the quality of the coal may be improved by excluding harmful bands, or how particular sections may be put to specially valuable uses. The regional variation of the seams has been traced in detail, and the ground securely laid for a forecast of the changes likely to be encountered as the seams are worked into new areas. Valuable evidence of the variation of coals in the vertical sequence has been obtained by the examination of the series of seams encountered in the exploratory borings for oil.

Following the nationalization of the coal reserves, a rapid survey was undertaken so that a final assessment might be based not only on the quantity but also on a carefully defined estimate of the qualities of the coals likely to be worked in the various royalties during the 'valuation period' (usually a hundred years). The coals were divided into six classes defined by volatile yield and coking properties, and the whole results finally subjected to mechanical analysis so as to reveal the total amounts of each group likely to be worked. This could be compared with the estimated reserves of each type, and led to the 
satisfactory conclusion that no type is likely to be exhausted during the next century.

The work of the organisation has naturally been forced to branch in many directions as ever-growing demands have been made upon it. Much work has been devoted to examination and advice related to the open-cast coal developments. Problems concerning the sizing, cleaning and breakage of coal have been investigated. Weathering and storage have been studied, and methods developed for the preservation of coal-dumps with tar cover. The selection and treatment of coals to serve producer-gas units for road-transport vehicles was undertaken during the War, as also the selection of coals to produce active earbon. The loss of the normal sources of sulphur led to an active search for pyritic waste from coal, and the production of 33,000 tons of sulphuric acid from this source. The accumulated knowledge and experience were also utilized for the solution of problems for the Service departments. For the future, the work of this organisation must be of the highest value for the proper planning of coal production and for its most economic and profitable use by the consumer.

As an indication of the need for intensive geological study and practical exploration, Dr. G. H. Mitchell submitted a contribution concerning the problem as it affects the Midland coalfields of Coalbrookdale, the Wyre Forest, South Staffordshire, Warwickshire, South Derbyshire and Leicestershire. These are parts of a natural area of related coalfields containing seams of a limited range of low- and medium-rank coals. The industry and the resultant centres of dense population within the area are founded on these coals, and especially on those of better quality. Within the life-time of the next generation there must be a steady decline in output unless new fields are discovered. Even sooner, the deterioration in average quality as a result of the exhaustion of the better seams will become a serious problem. The matter is therefore urgent.

The geological sequence within each of the fields is fairly completely known. Evidence for the estimation of their mutual relations is available in the marine beds and mussel bands which have been traced from field to field. The actual position, extent and depth of such extensions of these fields as may still lie concealed below the younger rocks of the intervening areas may be doubtfully inferred as the result of intensive geological study. Exhaustive investigation of the evidence of Carboniferous and postCarboniferous earth-movements, and of the consequent sedimentation and periods of erosion, must form the basis of a forecast. But results can be no more than probabilities and are inadequate for practical development. They can, however, suggest where borings may be located so as to yield the most valuable information. A programme of intensive work, in which boring and geological study proceed hand in hand, is likely to produce practical results at a cost quite infinitesimal in relation to the national importance of the matters involved. Since, however, the complexity of the problerns we have now to face in the search for new areas of coal is greater than those hitherto encountered, the boring and exploration programme must needs be far more extensive than any previously undertaken.

Dr. G. M. Lees delivered a communication prepared in conjunetion with L. N. Falcon on the relation of the search for oil to the discovery of new coalfields. One considerable buried field has already been proved in central Lincolnshire. Although too deep for present-day exploitation, there is good prospect that it may be at a lesser depth near the Wash. Farther south-eastward, extension of the Nottinghamshire coalfield has also been proved, with the possibility that it may link up with the Wash area. It is a remarkable fact that the Kent coalfield should be the sole example of a buried field hitherto located in Great Britain.

The search for oil has to commence from speculative geological deduction based on the limited surface evidence. Since the rocks and structures concerned are for the most part buried under unconformable cover, the most widespread indications, gathered from the Continent of Europe as well as from Britain, must be brought under review. Selected areas of sufficient promise are then subjected to gravity and magnetic survey to gain a broad generalized picture of the underground structure. From this background the most likely areas are more closely examined by seismic survey, and finally, where indications seem favourable, proved by boring.

The Midlands and south-central England have been generally studied. Of the areas selected within that region, one around Market Drayton and between there and Burton-on-Trent has been subjected to seismic survey. Boring will shortly be commenced. In three other areas, gravity and magnetic survey have been in progress for some twenty months, and results are now being analysed. These are: (1) an area in Wiltshire, where an eastward extension of the Somerset coalfield, or possibly an independent field, may be proved; (2) the Cotswold country in Gloucestershire, and perhaps southern Worcestershire ; and (3) certain areas in Oxfordshire, possibly between Oxford and Reading. In addition to these, eastern Essex has been indicated for further study, while numerous other areas exist outside the present programme of investigation where extensions of existing fields or new fields may be found.

Dr. Le日s concluded with special emphasis on the need for national effort in this matter. The present programme of private boring can only be subsidiary to the search for oil and at any time may have to be abandoned. Such a possibility is scarcely to be dispassionately contemplated.

The last contribution, from Dr. R. Holroyd, was on coal as a chemical raw material. Chemical industry is largely dependent on the availability of cokingcoals ; first, for the phenol, cresol, naphthalene and anthracene extracted from the two million tons of coal tar produced annually by coal carbonization, as well as benzene, toluene, xylene, etc.; and secondly, for the production of water-gas or hydrogen for chemical synthesis from about one million tons of coke with high melting-point ash.

The yield of aromatic raw materials is only about 1.5 per cent of the weight of the coal. In the past, this output of by-product by the carbonization industry has sufficed for chemical needs. Now, the anticipated demand greatly exceeds the supply, and investigation has therefore been directed to other sources. Considerable progress has been made in the development of petroleum-cracking plants, and it is probable that the chemical industry in future will be far less dependent on carbonization by-products, though it will continue to provide an outlet for them in so far as they can be produced economically.

The demand for 'synthetic gas' (carbon monoxide and hydrogen or hydrogen alone) is likely to increase considerably. The present high cost has compelled close examination of the efficiency of existing methods 
for its production and the investigation of new methods. A provisional scheme for a continuous process based on the 'fluid-solid' technique was described as offering considerable promise. German methods using oxygen may also be developed. While increased efficiency and lower costs must be the primary objective, it is likely that an even more important outcome may be the possibility of using a wider range of coals.

Thus it would appear that the chemical industry in future may be less exacting in its demands regarding types of coal and may obtain a large proportion of its raw materials from other sources. At the same time, it must remain a major consumer of coal as fuel, and its prosperity will depend on ample supplies.

George HICKLING

\section{BRITISH NON-FERROUS METALS RESEARCH ASSOCIATION}

\begin{abstract}
$\triangle$ FTER the annual general meeting of the British Non-Ferrous Metals Research Association on July 10, members and a small number of visitors were shown the laboratory buildings and facilities which the Association now possesses.

In 1930 the first of the Association's laboratories was established by conversion of a small factory near Euston Station, London, and just prior to the War additional laboratories were built on an adjacent site. During the War the original laboratory block was destroyed by enemy action, although the new building was fortunately undamaged. In preparation for postwar expansion, adjacent premises were afterwards purchased; their conversion is now almost completed, and application has been made for a licence to rebuild the war-damaged property. Completion of the present plans will provide a series of laboratories with a total of about 32,000 sq. ft. of floor space in an accessible position in central London. The extension of the laboratories and the corresponding increase in scientific staff, which together will enable a wider range of metallurgical work to be undertaken, have been made possible by the steady and continuing increase in the financial support which the Association receives from industry.
\end{abstract}

Expansion of the laboratory facilities was particularly required in the Physics, Mechanical Testing and Corrosion Sections, and now accommodation was needed for the recently formed Metal Finishing Section and to house metal-working equipment. Such metal-working equipment will be installed as is needed to prepare experimental alloys in wrought form for test purposes, but it is not intended for fundamental research on the working behaviour of metals and alloys, as it is hoped that such work will be done co-operatively with the British Iron and Steel Research Association in the latter's projected Flow of Metals Research Laboratory.

In accordance with the Association's policy of developing and using modern physical methods for the examination of metals, a Metropolitan-Vickers electron microscope has recently been installed in the physics laboratory and a study is being made of existing replica techniques. Attention will also be paid to the possibility of developing new replica techniques and alternative methods of examining metals in this instrument. Its full potentialities in metallurgical research have not yet been assessed, as its successful use depends so much on the develop- ment of satisfactory techniques, but a critical experimental survey is being made of its possible field of use so that members can be suitably advised. The high-tension source of the electron microscope will also be used for electron diffraction work, apparatus for which is being built in the Association's workshops. The Association's pioneering work of many years ago in the application of the spectrograph to the quantitative analysis of non-ferrous metals and alloys is being continued by a systematic study of the use of multiplier photocells in recording spectra and for investigation of excitation conditions.

The expansion of the Mechanical Testing Laboratory is largely to enable more work on creep and fatigue testing to be carried out, including fatigue testing at elevated temperatures, while the extension of the Corrosion Laboratories is called for by the great demands which are made on the Association to-day for further work in the field of corrosion, including stress corrosion.

Simultaneously with the re-planning and extension of its laboratories, the Association has recently reconsidered thoroughly its field of work and the organisation of its activities in relation to the needs of the industry it represents.

The non-ferrous metals industry is a varied group of industries comprising producers, manufacturers and users of the main industrial metals other than iron and steel. It includes a large number of firms with well-equipped and staffed research laboratories, and a number of small firms with little or no research facilities or technical staff of their own. The needs of the members, therefore, vary widely, and the Association's policy is first to carry out long-term researches designed to provide a basic understanding of the factors involved in the fabrication or service life of the materials in question, and secondly to help members, particularly the smaller ones, in the utilization of existing metallurgical knowledge in dealing with their immediate difficulties and in raising the technical efficiency of the industry.

The long-term research programme requires primarily a first-class scientific staff with all the equipment and facilities necessary for their work, but equally important is an adequate knowledge of the technology of the industry. The Association's staff is not by itself in a position to conceive and formulate the long-term projects which should be investigated, and for this purpose several industry research committees have been established consisting of the most highly qualified men of science available in the different sections of the industry. These committees are responsible, in co-operation with the staff, for the formulation of the research programme and for the appointment of sub-committees of industrial technicians, intimately acquainted with each particular problem, for the guidance and advice of the Association's staff in carrying out the work. These committees, of course, do not direct the research work in detail, which responsibility lies with the Director and senior staff, but serve to keep the staff fully informed on the industrial experience which is helpful during the course of the investigation.

At the other extreme, the problems of the small firm, which frequently needs information more than research, are dealt with by the Liaison Department with the backing of a comprehensive Library and Information Service. The Association's liaison staff maintains contact with the industry and with the research work in progress in the Association and elsewhere, and offers confidential service which is 